\title{
Epidemiology of dizziness in northern Poland - The first Polish neurootologic survey of the general population
}

\author{
Romuald Wojtczak ${ }^{1}$, Waldemar Narożny ${ }^{2}$, Jerzy Kuczkowski ${ }^{2}$, Janusz Siebert ${ }^{3}$ \\ ${ }^{1}$ Family Health Centre "Salus", Bytów, Poland \\ ${ }^{2}$ Department of Otolaryngology, Medical University of Gdańsk, Poland \\ ${ }^{3}$ Department of Family Medicine, Medical University of Gdańsk, Poland
}

Wojtczak R, Narożny W, Kuczkowski J, Siebert J. Epidemiology of dizziness in northern Poland - The first Polish neurootologic survey of the general population. Ann Agric Environ Med. 2017; 24(3): 502-506. doi: 10.5604/12321966.1228401

\begin{abstract}
Introduction. Dizziness is a common complaint among patients seen by primary care physicians, neurologist and otolaryngologist. It presents difficult clinical and diagnostic problems.

Objectives. The purpose of this study was to determine the prevalence and incidence of dizziness in the general population of the town and district Bytów, northern Poland, and to describe its clinical characteristics.

Materials and method. Epidemiological questionnaires were completed by 4,799 inhabitants of the town and district of Bytów. All participants were randomly selected from the patients' list of the Family Health Centre 'Salus' in Bytów. The main variables studied were gender, age, school education, work situation, clinical characteristics of dizziness symptoms and circumstances provoking dizziness.

Results. Prevalence of dizziness was diagnosed among $16.4 \%$ of the inhabitants of the town and district of Bytów. Symptoms of dizziness appeared more often among the elderly and among women. Among young people, the condition shows as vertigo or presyncope, among people aged 50 or older, the condition showed as disequilibrium. The most common precipitant of dizziness was postural change. Dizziness was more likely to occur among those suffering from high blood pressure, diabetes, increased blood lipids, hypothyroidism, cardiac arrhythmia and depression. Dizziness was more likely to occur among women using OCS or HRT, and individuals taking anti-epileptic drugs.

Conclusion. The authors present the first Polish neurootologic survey of epidemiology of dizziness in the general population. This condition is common in the general population, and this study indicates its prevalence in $16.4 \%$ of the inhabitants of the town and district of Bytów. It is recommendable that epidemiological studies should be carried out.
\end{abstract}

\section{Key words}

dizziness, general population, epidemiology, prevalence, neurology

\section{INTRODUCTION}

Dizziness is a non-specific term that describes a sensation of altered orientation in space.

Dizziness presents a challenge to clinicians and researchers due to its subjective nature. It is difficult to define, impossible to measure, a challenge to diagnose and troublesome to treat. It is ranked among the most common complaints in primary care office practice, affecting approximately $6.1 \%-21 \%$ of the general population $[1,2,3]$. Its prevalence increases with age and may be as high as $30 \%$ in those over 65 years of age [4]. Vertiginous syndromes account for $2 \%$ of consultations in general practice [5]. In many instances the symptoms resolve spontaneously, and the mortality and morbidity rate is low [6]. However, in $20 \%-40 \%$ of patients suffering from dizziness in primary care, the underlying cause of dizziness remains unknown [7].

In 1972, Drachman et al. described 4 types of dizziness: vertigo, presyncope, disequilibrium and lightheadedness [8]. Nearly 40 years later, for majority of researchers this typology remains the basis for the definition and classification of dizziness.

Address for correspondence: Waldemar Narożny, Department of Otolaryngology, Medical University of Gdańsk, Poland

E-mail: naroznyw@wp.pl

Received: 8 December 2012; accepted: 9 September 2014; first published:December 2016

\section{OBJECTIVES}

The aims of this study are to establish the frequency of dizziness occurrence among inhabitants of the town and district of Bytów in northern Poland, and attempting to establish the most important clinical characteristics of subtypes of dizziness reported by individuals living in this part of Poland.

\section{MATERIALS AND METHOD}

Epidemiological surveys were conducted between July September 2010 among 4,799 inhabitants of the town and district of Bytów, who were randomly selected from among patients of the 'Salus' Family Health Centre in Bytów. The surveys were conducted by means of an epidemiological questionnaire prepared for this purpose.

The questionnaire consisted of 4 parts. The first part of the questionnaire concerned demographic data, such as age, gender, education and work related situation. In the second part of the questionnaire, the respondents gave information about whether or not vertigo and balance disorders had occurred on one occasion in the period of 12 months prior to filling in the questionnaire. This was done by the means of criteria of dizziness, supplied in the questionnaire, as defined by Drachman et al. [8]: Vertigo is a suddenly appearing 
subjective sensation of movement (spinning, swinging, lifting,) of the outside world compared to the body or the body to the outside world, without any external reason for this movement; nausea and vomiting possible, as well as unsteadiness while walking.

Presyncope is a sensation close to loosing consciousness, collapsing and experiencing scotoma in the process, limited visibility, sensation of 'ringing' in ears, paleness of skin, sweating, nausea, sensation of weakness in the legs, as if they were made of jelly, but without loosing consciousness.

Disequilibrium is a postural instability described as involving legs and trunk without sensation in the head.

Lightheadedness is an insensibility, sinking in, disorientation, sensation of heavy head, fear of falling, sensation of instability, swaying ground.

Respondents who reported suffering from vertigo and balance disorders, selected one of 4 subtypes of dizziness. In the third part of the questionnaire, respondents who experienced vertigo and balance disorders, named the characteristics of their symptoms, and possible factors that caused them. In fourth and last part of the questionnaire, all respondents gave information about, among others, their eating habits, past and present conditions, and results of additional medical tests carried out up to that point in time.

The surveys were conducted by 6 pollsters trained to carry out the task, and were health care professionals. The questionnaires were filled in by respondents in the presence of the pollsters. Their task was to assist the respondents with any additional queries related to questions in the questionnaire. Respondents filled in the questionnaires in a room assigned and adapted for the purpose situated within the 'Salus' Family Health Centre in Bytów.

Statistical analysis was carried out using SPSS (Statistical Package for the Social Sciences), version 14.0 for Windows XP. A database of variables was created to reflect all variables from the research tool (the questionnaire). Altogether, 4,799 questionnaires were filled in. All presented results underwent an analysis of relationships between variables, using a chisquare test for the purpose, with the aim of comparing observed frequencies with expected frequencies, and to verify if relationships existed. In order to assess the level of statistical significance, C Pearson's and V Cramer's tests were used on the nominal scale level. Any value amounting to $\mathrm{p}<0.05$ was considered statistically significant.

\section{RESULTS}

Epidemiological surveys were conducted among 4,799 inhabitants of the town and district of Bytów who constituted $25.2 \%$ of the entire adult population of this region. No statistically significant differences were established between the group of people who took part in the study and the entire population of the town and district of Bytow ( $p>0.05)$. The group who took part in the study was representative of the general population of the town and district of Bytow.

During the 3-month study, each of the 4,799 inhabitants of the town and district Bytów completed questionnaires (100.0\%). Of the 4,799 respondents, 780 (16.4\%) reported experiencing dizziness, whereas the remaining 4,019 respondents (83.6\%) did not report such problems.

Patients' demographic and clinical characteristics are shown in Table 1, showing statistically significant relationships, and Table 2 presenting statistically insignificant relationships. The prevalence of dizziness was considerably higher in women than men $-76.2 \%$ ( $n-594)$ vs. $23.8 \%$ (n 186), respectively. The age of the patients belonging to the group suffering from dizziness and the group of respondents without any such condition was analyzed. Patients with dizziness who were over 50 y.o. constituted $45 \%$ of this group (351 out of 780 people), whereas respondents without any such condition who were over 50 y.o. constituted only $22.2 \%$ (848 out of 4019 people).

Respondents who were over 50 y.o. mainly complained about dizziness (351 people - $45 \%$ ), whereas the majority of the group who did not suffer from dizziness constituted young people who were less than 34 y.o. (2355 people $52.6 \%)$. It was concluded that statistically, the occurrence of dizziness was more frequent among people suffering from hypertension (22.1\% vs. $6.4 \%)$, increased blood lipids $(15.5 \%$ vs. $5.4 \%)$, diabetes (7.9\% vs. $2.8 \%)$, cardiac arrhythmia $(25.9 \%$ vs. $7.5 \%)$, hypothyroidism ( $4.1 \%$ vs. $1.3 \%)$, individuals who take anti-epileptic drugs ( $3.0 \%$ vs. $0.7 \%$ ), women using OCS (4.1\% vs. $1.0 \%)$. A strong association was found between dizziness with self-reported depression (22.1\% vs. $6.1 \%)$.

Table 1. Demographic and clinical characteristics of patients with and without dizziness - occurrence of statistically significant relationship $(p<0.05)$

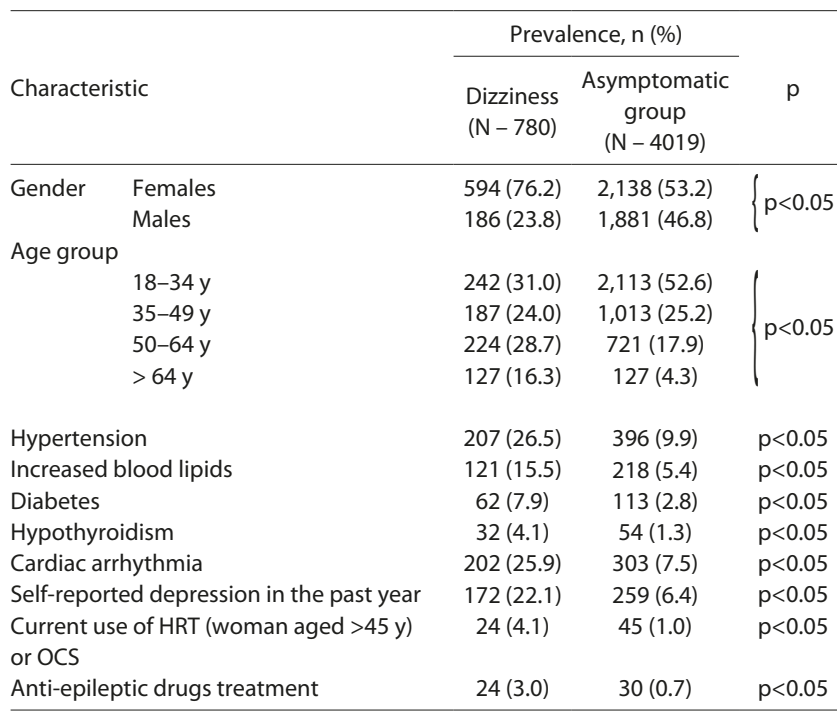

HRT - hormone replacement therapy; OCS - oral contraceptive; $p<0.05$ - statistically significant relationship; $\mathrm{n}$ - total number of patients; $\mathrm{N}$ - number of patients in analyzed groups

No statistically significant relationship was found between the 2 groups who took part in the survey (dizziness group vs. asymptomatic group) when the following factors were used as a point of reference: school education, work situation (active vs. retired), stroke, migraine, hyperthyroidism, motion sickness, drug use (antihistamines, anxiolitics/hypnotics, analgesics, diuretics, aminglicoside antibiotics, antimalarics, cytostatics), smoking, alcohol and salt abuse. It was noticed that patients suffering from dizziness more frequently experienced Migraines, compared to other patients who did not suffer from balance disorders (respectively, 34.9\% compared to $18.3 \%$ ).

The subtypes of dizziness found are shown in Table 3. The most common dizziness subtype was vertigo (41.1\%), followed by presyncope (38.5\%), disequilibrium (13.7\%) and lightheadedness (6.7\%). The age of patients was analyzed in 
Table 2. Demographic and clinical characteristics of patients with and without dizziness - no occurrence of statistically significant relationship $(\mathrm{p}>0.05)$

\begin{tabular}{|c|c|c|c|}
\hline \multirow[b]{2}{*}{ Characteristic } & \multicolumn{2}{|c|}{ Prevalence, n (\%) } & \multirow[b]{2}{*}{$\mathrm{p}$} \\
\hline & $\begin{array}{l}\text { Dizziness } \\
(\mathrm{N}-780)\end{array}$ & $\begin{array}{l}\text { Asymptomatic group } \\
\qquad(\mathrm{N}-4019)\end{array}$ & \\
\hline \multicolumn{4}{|l|}{ School education: } \\
\hline Higher level & $132(16.9)$ & $602(15.2)$ & \multirow{3}{*}{$p>0.05$} \\
\hline Middle level & $532(68.3)$ & $3,078(77.5)$ & \\
\hline Lower level & $115(14.8)$ & $292(7.4)$ & \\
\hline \multicolumn{4}{|l|}{ Work situation: } \\
\hline Active & $390(50.3)$ & $2,669(67.4)$ & \multirow{2}{*}{$p>0.05$} \\
\hline Retired & $386(49.7)$ & $1,291(32.6)$ & \\
\hline History of stroke & $17(2.2)$ & $36(0.8)$ & $p>0.05$ \\
\hline Migraine & $272(34.9)$ & $736(18.3)$ & $p>0.05$ \\
\hline Hyperthyroidism & $39(5.0)$ & $86(2.1)$ & $p>0.05$ \\
\hline Motion sickness & $112(14.4)$ & $399(9.9)$ & $p>0.05$ \\
\hline \multicolumn{4}{|l|}{ Drugs: } \\
\hline Antihistamines & $181(23.2)$ & $596(14.8)$ & $p>0.05$ \\
\hline Anxiolotics/hypnotics & $146(18.7)$ & $502(12.5)$ & $p>0.05$ \\
\hline Analgesics & $11(1.4)$ & $43(1.1)$ & $p>0.05$ \\
\hline Diuretics & $61(7.8)$ & $185(4.6)$ & $p>0.05$ \\
\hline Aminoglicoside antibiotics & $144(18.5)$ & $576(17.9)$ & $p>0.05$ \\
\hline Antimalarics & $3(0.4)$ & $7(0.2)$ & $p>0.05$ \\
\hline Cytostatics & $8(1.0)$ & $16(0.4)$ & $p>0.05$ \\
\hline Smoking (current daily habit) & $227(29.1)$ & $1,476(36.7)$ & $p>0.05$ \\
\hline Alcohol abuse & $113(2.8)$ & $17(2.2)$ & $p>0.05$ \\
\hline Salt abuse (> 6 g daily) & $221(28.3)$ & $1,086(27.0)$ & $p>0.05$ \\
\hline
\end{tabular}

$\mathrm{p}>0.05$ - statistically insignificant relationship; $\mathrm{n}$ - total number of patients; $\mathrm{N}$ - number of patients in analyzed groups

each of the subtypes of dizziness. Vertigo and presyncope occurred more frequently among young people who were less than 34 y.o. (respectively, $33.3 \%$ and $35.0 \%$ ). Disequilibrium was reported mainly by older respondents who were over 50 y.o. (72. $9 \%$ people suffering from the dizziness subtype). The least frequently reported subtype was lightheadedness (52/780).

Table 3. Subtypes of dizziness in 780 participants with dizziness

\begin{tabular}{|c|c|c|c|c|c|c|c|c|c|c|}
\hline \multirow{3}{*}{$\begin{array}{l}\text { Subtypes of } \\
\text { Dizziness }\end{array}$} & \multirow{2}{*}{\multicolumn{2}{|c|}{$\begin{array}{l}\text { Dizziness } \\
\text { group } \\
(n-780)\end{array}$}} & \multicolumn{8}{|c|}{ Age groups } \\
\hline & & & \multicolumn{2}{|c|}{$18-34 y$} & \multicolumn{2}{|c|}{$35-49 y$} & \multicolumn{2}{|c|}{$50-64$ y } & \multicolumn{2}{|c|}{$>65 y$} \\
\hline & $\mathrm{N}$ & $\%$ & $\mathrm{~N}$ & $\%$ & $\mathrm{~N}$ & $\%$ & $\mathrm{~N}$ & $\%$ & $\mathrm{~N}$ & $\%$ \\
\hline Vertigo & 321 & 41.1 & 107 & 33.3 & 97 & 30.2 & 91 & 28.4 & 26 & 8.1 \\
\hline Presyncope & 300 & 38.5 & 105 & 35.0 & 62 & 20.7 & 88 & 29.3 & 45 & 15.0 \\
\hline Disequilibrium & 107 & 13.7 & 12 & 11.2 & 17 & 15.9 & 36 & 33.6 & 42 & 39.3 \\
\hline Lightheadedness & 52 & 6.7 & 18 & 34.6 & 11 & 21.1 & 16 & 30.8 & 7 & 13.5 \\
\hline
\end{tabular}

$\mathrm{n}$ - total number of patients; $\mathrm{N}$ - number of patients in analyzed groups

Provoking circumstances are displayed in Table 4. The most common precipitant of dizziness was postural change (standing up - 49.0\%, changes in body position - $32.6 \%$ ), but head and neck movements were also frequently reported (rapid head movement - 43.5\%). Among 343 respondents (44.0\%), dizziness was precipitated by strong emotions or stress. Some of the patients reported more than one precipitant of dizziness.

\section{DISCUSSION}

Until now, only 3 epidemiological studies have been carried out to determine the incidence of dizziness in the general population. Nakashima et al. [2] conducted a postal survey in 1996 that reached 7,685 respondents, and concluded
Table 4. Provoking circumstances in 780 participants with dizziness

\begin{tabular}{lc} 
Provoking circumstances* & $\begin{array}{c}\text { Dizziness group } \\
(\mathrm{n}-780) \\
\mathrm{N} \%\end{array}$ \\
\hline Standing up & 38249.0 \\
Strong emotions, stress & 34344.0 \\
Rapid head movement & 43.5 \\
Changes in position of body (e.g. turning over in bed) & 32.6 \\
Time of day, particular seasons & 22.2 \\
Menstrual periods (if relevant) & $11920.0^{* *}$ \\
Loud noises & 15319.6 \\
Heat, hot shower & 11715.0 \\
Aeroplane, boat or car travel & 9912.7 \\
Coughing, blowing the nose, or straining & 7810.0 \\
Elevators & 7810.0 \\
Gym exercises & 607.7 \\
Walking in a dark room & 476.0 \\
No provoking circumstances & 455.8
\end{tabular}

* Adds up to more than $100 \%$, because more than one answer is possible

** Percentage for 594 women; $\mathrm{n}$ - total number of patients; $\mathrm{N}$ - number of patients in analyzed groups

that dizziness affected $6.1 \%$ of them. Neuhauser et al. [3] conducted a telephone survey in 2005 that reached 4,869 respondents, and concluded that $7.8 \%$ of the respondents suffered from dizziness. Hannaford et al. [1] conducted a postal survey in 2005 that reached 15,788 respondents, and concluded that $21.0 \%$ of respondents suffered from dizziness. The presented survey demonstrates that $16.4 \%$ out of 4,799 respondents and inhabitants of the town and district Bytów suffered from dizziness. This is the fourth survey in the world and the first in Poland to concentrate on this issue, and $n$ conducted among the general population. In the available publications, the authors of the current study did not find any instances of a survey carried out directly, in which the respondents had direct contact with a pollster.

The number of people suffering from dizziness increases with age. Sloane et al. [5] reported that problems occur in: $0.2 \%$ of patients aged $0-14 \mathrm{y}, 0.7 \%$ patients aged $15-25 \mathrm{y}, 1.8 \%$ patients aged $25-34 \mathrm{y}$, and $2.4 \%$ patients aged $45-54 \mathrm{y}$. It is the most frequently occurring complaint in patients aged 75 and over $[5,9]$. When 758 inhabitants of Göteborg in Sweden were consulted, Sixt et al. [10] concluded that dizziness was a health problem among $40 \%$ of females and $30 \%$ of males aged 75 or over. The authors conclusions were similar $[1,11]$. Falls, which accompany dizziness in people aged 65 or over, are the most dangerous results of the condition. The increase in the rate of falls in people who are at the age specified above and live in their own household, is estimated at $20-40 \%$, whereas this rate in care homes might double $[12,13]$. The current conclusion on the increase in the number of people suffering from dizziness with age is in compliance with data presented in publications on this subject.

The majority of epidemiology studies focusing on dizziness conclude that there are more occurrences among women. Such a difference was concluded by: Neuhauser et al. [3] (54\% vs. $46 \%$ ), Hannaford et al. [1] (52\% vs. $48 \%$ ), Garrigues et al. [14] (69\% vs. 31\%).

Research by the authors of the presented study indicate that the occurrence of dizziness among women is 3 times higher than in men (76.2\% vs. $23.8 \%)$. Among factors that could explain this phenomenon are: stronger relationship between migraine and dizziness among women than men, and the impact of hormonal factors. 
Results of epidemiological studies conducted to-date on the link between dizziness and conditions such as heart and vascular disease, cerebrovascular disease, hormonal disorders, metabolic disorders, and personality disorders, are ambiguous. The presented study establishes that dizziness affects the following groups more frequently to a statistically significant level: people with hypertension $(22.1 \%$ vs. $6.4 \%)$, increased blood lipids ( $15.5 \%$ vs. 5.4\%), diabetes (7.9\% vs. $2.8 \%)$, cardiac arrythmia ( $25.9 \%$ vs. $7.5 \%)$, hypothyroidism $(4.1 \%$ vs. $1.3 \%)$, people taking anti-epileptic drugs $(3.0 \%$ vs. $0.7 \%)$, women using OCS or HRT (4.1\% vs. $1.0 \%)$ and people with self-reported depression (22.1\% vs. $6.1 \%)$. Maarsingh et al. [15] established that the most common major contributory cause of dizziness in 417 elderly patients in primary care was cardiovascular disease. These authors analyzed thoroughly and critically the surprising results of their study, and reached the conclusion that they might be a confirmation of an important phenomenon: investigators tend to diagnose conditions that they know about or are interested in. Research carried out by Colledge et al. [16] constitutes an example. They performed magnetic resonance imaging of the head and neck and reported an unusually high prevalence of cervical spondylosis $(66 \%)$ as a cause of dizziness. Sloane et al. [17] referred to this phenomenon as 'blind men and the elephant'.

The most frequently occurring subtype of dizziness treated by general practitioners is vertigo. Patients suffering from this condition, as per analyzed material, amounted to the following percentage of patients: Hanley et al. [18] - 41.2\%, Bird et al. [19] - 32\%. Symptoms of the following were less frequent: lightheadedness (respectively, 30.5\% and 12.0\%) and disequilibrium (respectively, $14.7 \%$ and $4.0 \%$ ). Determining the subtype of dizziness was not possible with all patients (Hanley et al. [18] - 7.1\%, Bird et al. [19] - 41.0\%). Frequency of subtypes of dizziness in patients aged 65 or over varies. As per Colledge et al. [4], patients with disequilibrium were a majority (42.0\%); the following were less frequent: vertigo (32.0\%) and lightheadedness (21.0\%). Maarsingh et al. [15] concluded that in a group of 417 patients suffering from dizziness (aged 65 or over), 69\% reported symptoms of presyncope. The current research concluded that in a group of 780 patients suffering from dizziness, the most frequent subtypes were vertigo (41.1\%) and presyncope (38.5\%). Vertigo and presyncope were found more often among young people aged 34 or less (among $33.3 \%$ and $35.0 \%$ of respondents, respectively). Disequilibrium was found mainly among the older population aged 50 or over (72.9\%).

Among the majority of inhabitants of the town and district Bytów, precipitants of dizziness were changes in body position (e.g. turning over in bed, standing up) and rapid head movement. Colledge et al. [4] listed as the most frequent precipitants: rising from lying to standing - $42 \%$, bending over $-42 \%$, turning the head - $29 \%$, looking up $29 \%$, walking $-15 \%$.

Data exchange between research centres and researchers working on neurootological issues is hindered due to lack of commonly accepted classification of vertigo and balance disorders, and differences between terminology in use. In Europe, the following classification established by Drachman et al. [8] is nearly commonly applied: distinction of dizziness into vertigo, presyncope, disequilibrium and lightheadedness. Outside Europe, distinction of vertigo into vestibular vertigo and non-vestibular (disequilibrium and dizziness) is more commonly used. According to Maarsingh et al. [15], the results presented in studies can be influenced by different semantic and cultural meanings of the word 'dizziness' in English and national languages of authors. Even when studied in a single, English-speaking country, the term 'vertigo' has been shown to have diverse meanings for patients, general physicians, and even otologists. The decision taken by the Bárány Society in 2006, to promote the development of an implementable classification of vestibular disorders is a ray of hope. As a result of this decision, the Committee for the Classification of Vestibular Disorders of the Bárány Society started its work and published its first consensus document in 2009 [20].

\section{CONCLUSIONS}

The authors present the first Polish neurootologic survey of the general population on epidemiology of dizziness. This condition is common in the general population, and the presented indicates its prevalence in $16.4 \%$ of the inhabitants of the town and district of Bytów. Dizziness always presents difficult diagnostic and clinical problems. It is recommended that further extensive and broad-based epidemiological studies should be carried out on the distribution, determinants and outcome of dizziness. This would facilitate clinical decision-making and can help in understanding the underlying causes of dizziness.

\section{Acknowledgment}

This work was supported by the State Committee for Scientific Research ST72 (Medical Univeristy of Gdańsk, Poland)

\section{REFERENCES}

1. Hannaford PC, Simpson JA, Bissot AF, Davis A, McKerrow W, Mills $\mathrm{R}$. The prevalence of ear, nose and throat problems in the community: results from a national cross-sectional postal survey in Scotland. Fam Pract. 2005; 22: 227-233.

2. Nakashima K, Yokoyama Y, R. Shimoyama R, et al. Prevalence of neurologic disorders in Japanese town. Neuroepidemiology 1996; 15: 208-213.

3. Neuhauser HK, Brevern M, A. Radtke A, Lezius F, Feldman M, Ziese T, et al. Epidemiology of vestibular vertigo. A neurotologic survey of the general population. Neurology 2005; 65: 898-904.

4. Colledge NR, Barr-Hamilton RM, Lewis SJ, Sellar RJ, Wilson JA. Evaluation of investigations to diagnose the cause of dizziness in eldery people: a community based controlled study. BMJ 1996; 313(7060): 788-792.

5. Sloane PD. Dizziness in primary care. Results from National Ambulatory Medical Care Survey. J Fam Pract. 1989; 29(1): 33-38.

6. Sloane PD, Dallara J, Roach C, Bailey KE, Mitchell M, McNutt R. Management of dizziness in primary care. J Am Board Fam Pract. 1994; 7: 1-8.

7. Madlon-Kay DJ. Evaluation and outcome of the dizzy patient. J Fam Pract. 1985; 21(2): 109-113.

8. Drachman DA, C.W. Hart CW. An approach to the dizzy patient. Neurology 1972; 22(4): 323-334.

9. Sloane PD, Coeytaux RR, Beck RS, Dallara J. Dizziness: state of the science. Ann Intern Med 2001; 134: 823-832.

10. Sixt E, Landahl S. Postural disturbances in a 75-year-old population: I. Prevalence and functional consequences. Age Ageing 1987; 16: 393-398.

11. Grimley EJ. Transient neurological dysfunction and risk of stroke in elderly English population: the different significance of vertigo and non-rotatory dizziness. Age Ageing 1990; 19: 43-49.

12. Perry B. Falls among the aged living in a high-rise apartments. J Fam Pract. 1982; 14: 1069-1073. 
13. Tinetti ME. Factors associated with serious injury during falls by ambulatory nursing home residents. J Am Geriatr Soc. 1987; 35 (7): 644-648.

14. Garrigues HP, Andres C, A. Arbaizar A, Cerdan C, Meneu V, Oltra JA et al. Epidemiological aspects of vertigo in general population of the Autonomic Region of Valencia, Spain. Acta Otolaryngol. 2008; 128: 43-47.

15. Maarsingh OR, Dros J, Schellevis FG, Weert HC, Windt DA, Riet G et al. Causes of persistent dizziness in elderly patients in primary care. Ann Fam Med. 2010; 8(3): 196-205.

16. Colledge NR, Wilson JA, Macintyre CCA, MacLennan WJ. The prevalence and characteristics of dizziness in an elderly community. Age Aging 1994; 23: 117-120.
17. Sloane PD, Dallara J. Clinical research and geriatric dizziness: the blind med and the elephant. J Am Geriatr Soc. 1999; 47 (1): 113-114.

18. Hanley K, O'Dowd T. Symptoms of vertigo in general practice: a prospective study of diagnosis. Br J Gen Pract. 2002; 52: 809-812.

19. Bird JC, Beynon GJ, Prevost AT, D. Baguley DM. An analysis of referral patterns for dizziness in the primary care setting. Br J Gen Pract. 1998; 48: $1828-1832$.

20. Bisdorff A, Brevern M, Lempert T, Newman-Toker DE. Classification of vestibular symptoms: toward an international classification of vestibular disorders. Int J Vest Res. 2009; 19: 1-13. 